\title{
MEDICAL IMAGES COMPRESSION BASED ON SPIHT AND BAT INSPIRED ALGORITHMS
}

\author{
Sarah Saadoon Jasim ${ }^{1}$ \\ ${ }^{1}$ Middle Technical University, Computer Science/Technical college of Management, \\ Baghdad/Dept. of IT, Iraq
}

\begin{abstract}
There is a significant necessity to compress the medical images for the purposes of communication and storage. Most currently available compression techniques produce an extremely high compression ratio with a high-quality loss. In medical applications, the diagnostically significant regions (interest region) should have a high image quality. Therefore, it is preferable to compress the interest regions by utilizing the Lossless compression techniques, whilst the diagnostically lessersignificant regions (non-interest region) can be compressed by utilizing the Lossy compression techniques. In this paper, a hybrid technique of Set Partition in Hierarchical Tree (SPIHT) and Bat inspired algorithms have been utilized for Lossless compression the interest region, and the non-interest region is loosely compressed with the Discrete Cosine Transform (DCT) technique. The experimental results present that the proposed hybrid technique enhances the compression performance and ratio. Also, the utilization of DCT increases compression performance with low computational complexity.
\end{abstract}

Keywords: Medical images Compression, SPIHT Algorithm, Bat Inspired Algorithm, DCT.

\section{INTRODUCTION}

It is a very important requirement that any image being fastened and stored for fast transmission and storage because of small bandwidth, power consumption and so on. Particularly, in the case of medical applications, the number of architectures to be used for image compression must be simple and efficient. Today, the most important and effective coding methods used for image compression are Embedded Zerotrees of Wavelet transforms (EZW), Set Partition in Hierarchical Tree (SPIHT) and Embedded Block Coding with Optimized Truncation (EBCOT) algorithms are included. Today's JPEG image compression standard is based on these algorithms. In the JPEG standard, previously, Discrete Cosine Transform (DCT) was used, but it was replaced by Discrete Wavelet Transform (DWT), problems such as increased compression success and block distortions have been eliminated [1].

SPIHT is one of the most common and widely used coding algorithms. The SPIHT image coding algorithm uses the feature of the sub-band resemblance between the subbands and the fact that the high frequency sub-bands often contain negligible coefficients. It is easy and highly efficient in terms of coding output. It is an efficient and improved form of EZW algorithm. The algorithm provides a good quality copy of the image compressed by a small amount of data transmission. The SPIHT algorithm performs the same or better performance as EZW, but does not require an arithmetic encoder. As the arithmetic encoder hardware is costly and complex in cost, SPIHT provides cheaper and faster software solutions.
As a state of the art image compression technique, the SPIHT clearly has a variety of applications. N.R. Rema et al. [2] presented a modified SPIHT technique, suitable for mobile application for compressing color and grayscale images with a good Peak Signal to Noise Ratio (PSNR) performance. For medical applications, there are lots of researchers, C. Kamargaonkar and M. Sharma [3] proposed a hybrid compression technique in which the SPIHT algorithm has been utilized for Lossless compression the interest region, and the non-interest region is loosely compressed with the Haar wavelet transform. This technique presents an average of PSNR value equal to $41 \mathrm{~dB}$ for medical images. M. Sharma et al. [4] proposed a lossless compression technique based on SPIHT and motion vector with better values of PSNR for medical images. The motion vector is compressing the motion among the sequences of adjacent image. Another compression technique based on SPIHT has been presented by B. Panjavamam and P. T. V. Bhuvaneswari [5] for medical images. This technique proved that depending on the quality parameters values, the transmission bit rate can be selected for effective utilization of bandwidth, When the bit rate equal to 4 , the average of PSNR value is more than $53 \mathrm{~dB}$, and when the bit rate equal to 2 the average of PSNR value is more than $40 \mathrm{~dB}$.

In this paper, an efficient technique based on SPIHT and Bat inspired algorithms with a simple architecture has been utilized for image compression. The findings of the proposed technique and the success of the architecture have been evaluated with known objective criteria. The rest of the work can be summarized as follows; In the next section, the Bat and SPIHT algorithms have been described, the proposed compression technique is presented in the 3rd section, and the findings are shown in the 4 th section. The conclusions are given in the final section.

\section{Preliminaries}

\section{A. Bat Search Algorithm}

Bat algorithm (BA) is a propelled calculation created by Yang in 2010 and it has been observed to be exceptionally proficient [6]. The standard bat calculation depended sonar characteristics of micro bats. Therefore, echolocation must be explained.

\section{1) Echolocation of Micro Bats}

There are around 1000 unique types of bats [7], their sizes can differ broadly, extending from the small honey bee bat of around to the goliath with of around weigh around. Uses echolocation to a specific every one of animal groups, miniaturized scale bats utilize echolocation broadly, while super bats don't. Echolocation is utilized to identify prey, and obtain their perching fissure oblivious. Small scale bats can radiate a noisy sound heartbeat and tune in for the reverberate 
that ricochets over from the encompassing articles [8]. Their heartbeats fluctuate in properties and can be related with their chasing systems, contingent upon the species. Most bats use short recurrence balanced signs to clear through around an octave, and each heartbeat keeps going a couple of a seconds (about 4-15) of these recurrence scope in $35 \mathrm{kz}$ to $130 \mathrm{kz}$. Normally small-scale bats can produce about 10-20 such stable blasts each second, and the rate of heartbeat discharge can be accelerated to around 200 heartbeats for every second. Because the sound speed in air is around $\mathrm{v}=333 \mathrm{~m}$, the wavelength $\lambda$ of the ultrasonic sound floods with an unfaltering repeat $\mathrm{f}$ is given by $\lambda=\mathrm{v} / \mathrm{f}$, that is in the extent of $3 \mathrm{~mm}$ to $12 \mathrm{~mm}$ for the normal repeat go $22-120 \mathrm{kHz}$. Strikingly the wavetable a similar request of bird tall. spite of the fact that truly small-scale bats can likewise utilize in day on ears and clamor varieties to detect 3-dim environment. A few highlights of the echolocation would some be able to interface them with the target capacity of an improvement issue, which makes it conceivable to detail a shrewd, bat algorithm.

\section{2) Bat Algorithm}

Bat inspired is built up with the accompanying three glorified standards: firstly; All bats utilize echolocation to identify partition, and they similarly 'know' the complexity between and establishment obstacles mysterious way; secondly, Bats fly indiscriminately with speed vi at position xi with a repeat $f_{\text {mins }}$, changing wavelength $\lambda$ for prey. They can thus change the wavelength (or repeat) of their created thumps surge $r \in$ $[0,1]$, dependent upon the region of their goal; Thirdly, Although the commotion can from different points of view, we acknowledge that the tumult from a tremendous (positive) A0 to a base unfaltering regard Amin.

For effortlessness, beam following in isn't utilized in this calculation, however it can shape an intriguing component for facilitate expansion. When all is said in done, beam following can be computational broad, yet it very well may be an exceptionally helpful component and different applications. Besides, give recurrence inherently connected to a wave size. For instance, recurrence scope on $[230 \mathrm{kH}, 510 \mathrm{kH}]$ relates scope wavelengths from $2.7 \mathrm{~mm}$ to $12 \mathrm{~mm}$ noticeable all around. Hence, the change can be depicted either as far as recurrence e to suit diverse app, contingent upon this simplicity on usage with different components. Each bat is related with a speed vt and an area xt, at emphasis e, in a fdimel pursuit of arrangement place. with every one of Bat, these exist present strong arrangement $\mathrm{q} *$. Along these lines, these over 3 standards converted refreshing conditions on $\mathrm{xt}$ and eet in Eq.s (1, 2, and 3).

$$
\begin{gathered}
f_{i}=f_{\text {min }}+\left(f_{\text {max }}-f_{\text {min }}\right) \beta \\
v_{i}^{t}=v_{i}^{t-1}+\left(x_{i}^{t-1}-x_{*}\right) f_{i} \\
x_{i}^{t}=x_{i}^{t-1}+v_{i}^{t}
\end{gathered}
$$

Then $\beta s \in[2,2]$ arbitrary dissemination. specified before, wavelengths or frequencies can be utilized for usage, fmin= 0 and $f \max =O(1)$, contingent upon the area size of the issue of intrigue. Firstly, very bat is haphazardly allotted a recurrence that is shown consistently from [ $\mathrm{f} \min , \mathrm{f} \max$ ]. Hence, bat calculation can be represented as a recurrence tuning calculation for giving an adjusted blend of investigation and abuse. The clamor and heartbeat emanation rates basically give a component to programmed management and auto bigger locale with founding arrangements [6]. Each bat is encoded with a speed vt and an area $\mathrm{xt}$, at cycle e, in a r-dim pursuit arrangement. This area be considered an answer vector to an issue of intrigue (see Algorithm 1).

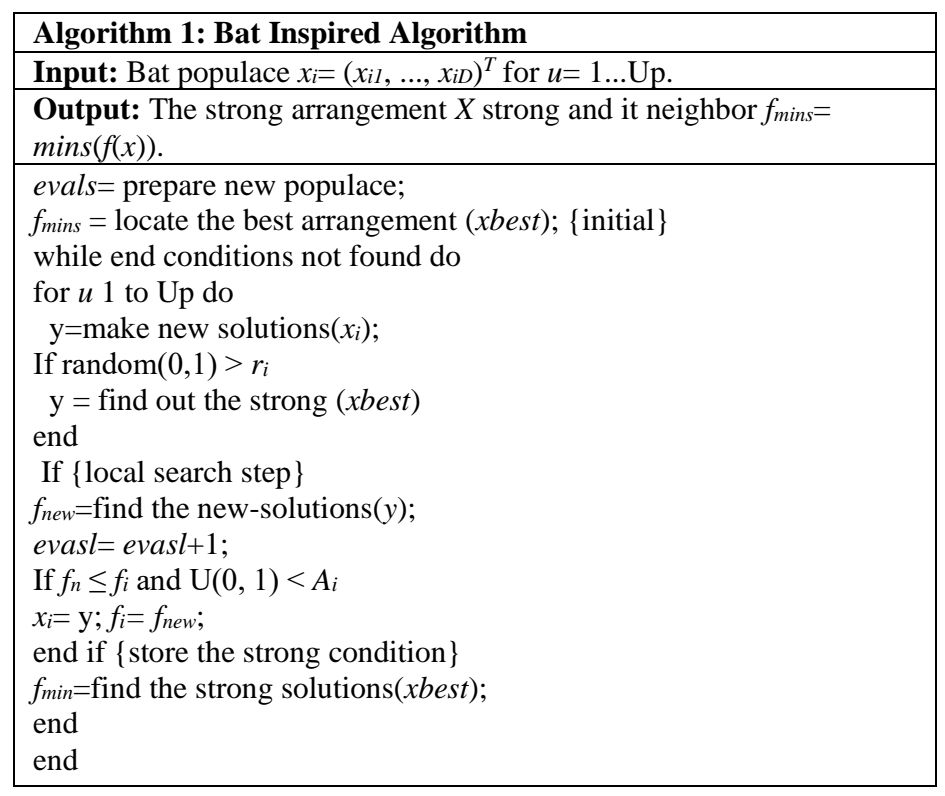

\section{B. SPIHT Algorithm}

This algorithm is developed regarding to the embedded zero tree wavelet (EZW) coding approach. It is derived from the wavelet transform-Haar type, which contains HH, LH, HL, LL and sub-bands. It exploits the spatial orientation trees then set the partitioning sorting algorithm. The SPIHT performs relationships of parent-children between the sub-bands to create a spatial orientation tree (SOT). The SOT structure is depicted in Figure 1, where its coefficients are distributed on three sub-bands HH, LH, and LL. The LL sub-band coefficients are arranged in $2 \times 2$ blocks. Each block coefficients do not relative to other coefficients nor join any tree. Meanwhile, the coefficients of top-right side are parent and adjacent to the coefficients of HL sub-band, which located in the same position of the LL sub-band. In addition, the bottom-right side is parent for corresponding coefficients in the adjacent $\mathrm{HH}$ sub-band, and so on [9].
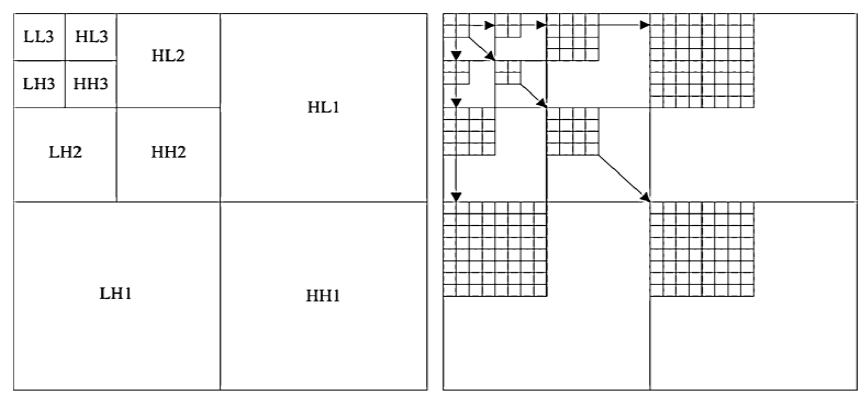
Fig 1: The Structure of Parent-Child of the wavelet coefficients according to SPIHT algorithm [9].

The lower levels are named children, which are parent to 4coefficients in same spatial position of HH, HL, and LL subbands. Each tree in the structure contains either HH, HL, or LL coefficients not mixture. The tree or branch of tree may contain on a set of pixels. When each sub-band has an $\mathrm{M} \times \mathrm{N}$ size, the algorithm will be constructed with $3 \mathrm{M} \times \mathrm{N} / 4$ trees, where 3 out of 4 sub-band coefficients is considered the tree root. Thus, the tree has coefficients of compressed spatial region, which contains all $\mathrm{HH}, \mathrm{HL}$, and LL sub-bands coefficients that is derived from this region. Therefore, the sub-bands have features that are known as self-similarity, which are specially localized in image features. The lower frequency component is carried by the top of structure while the higher frequency component is carried by the bottom. Consequently, most of energy is conserved in the top of tree (sub-bands).

The necessary definitions of the SPIHT algorithm that are used in literature for location $(i, j)$ in some sub-band [9]:

$\mathrm{O}(\mathrm{i}, \mathrm{j})$ : It is a set of all offspring coordinates of location (i,j) (one level below in the spatial tree).

$D(i, j)$ : It is a set of all descendant coordinates of location $(i, j)$ (all levels below in the spatial tree).

$\mathrm{H}$ : It is a set of coordinates of all coefficients in LL sub-band.

$L(i, j)=D(i, j)-O(i, j)$. It is all the descendants of location $(i, j)$, but not including the direct offspring. The following equation is the key of this algorithm:

$$
S_{n}(T)=\left\{\begin{array}{lr}
1, & \max _{(i, j) \in T}\left\{\left|c_{i, j}\right|\right\} \geq 2^{n} \\
0, & \text { otherwise }
\end{array}\right.
$$

Where $\mathrm{T}$ denotes a set of coordinates, and $\mathrm{f}(.,$.$) is a certain$ coefficient at location ( $i, j)$. The set $\mathrm{T}$ is called significant according to quantization level $n$ when $\operatorname{Sn}(T)=1$ whereas is called insignificant elsewhere. When some pixel has been tested for significant, $\operatorname{Sn}(\{(i, j)\})$ is rewritten as $\operatorname{Sn}(i, j)$ for simplified notation. Three lists are used by the encoding process of the SPIHT algorithm:

- Insignificant Pixels List (LIP): It includes separatedcoefficients, which have magnitudes that smaller than the thresholds.

- Insignificant Sets List (LIS): It includes a set of wavelet-coefficients, which are known a tree structures and are obtained with magnitudes that smaller than the threshold.

- Significant Pixels List (LSP) - It is the pixels list, which are obtained with magnitudes that larger than the threshold.

The algorithm SPIHT contains the stages; initialization, sorting pass and refinement pass. The 1st stage evaluates the threshold regarding to maximum value of wavelet-coefficients. After that, the LSP list saves the coefficient coordinates in the coarsest-level of the pyramid algorithm (LL sub-band) in LSP list and those that have descendants to LIS list. In the 2nd stage, the elements of LIP and LIS lists are sorted. For the LIP list, every pixel is performed a significance test against the threshold and the test result (i.e., 0 or 1 ) is delivered to form the bit stream. Once the coefficient is a significance, it will be coded, and its coordinate will be moved to the LSP list. Through the process of the sorting pass stage, the SPIHT algorithm performs the significance test for every set in the LIS list and delivers the significance information (i.e., 0 or 1 ). When the resulting set is significant, it is divided into its offspring and leaves. Threshold is siplited into two and the second and third stages are reprocessed until the targeted bit rate has been achieved [9].

\section{The Proposed Hybrid CoMPression TeChNiQue}

In this paper, identifying and compressing for the interest region and non-interest region of the medical image have been applied. The proposed hybrid compression technique encompasses the compression and decompression processes. The compression process requires firstly splitting the medical images in to two types of areas; interest region and noninterest region, and secondly compressing the interest region by utilizing the SPIHT and Bat inspired algorithms, whilst the non-interest region has been compressed by utilizing the DCT technique. The overall framework of the proposed technique is illustrated in Figure 2. While the decompression process works on reconstructing the original image.

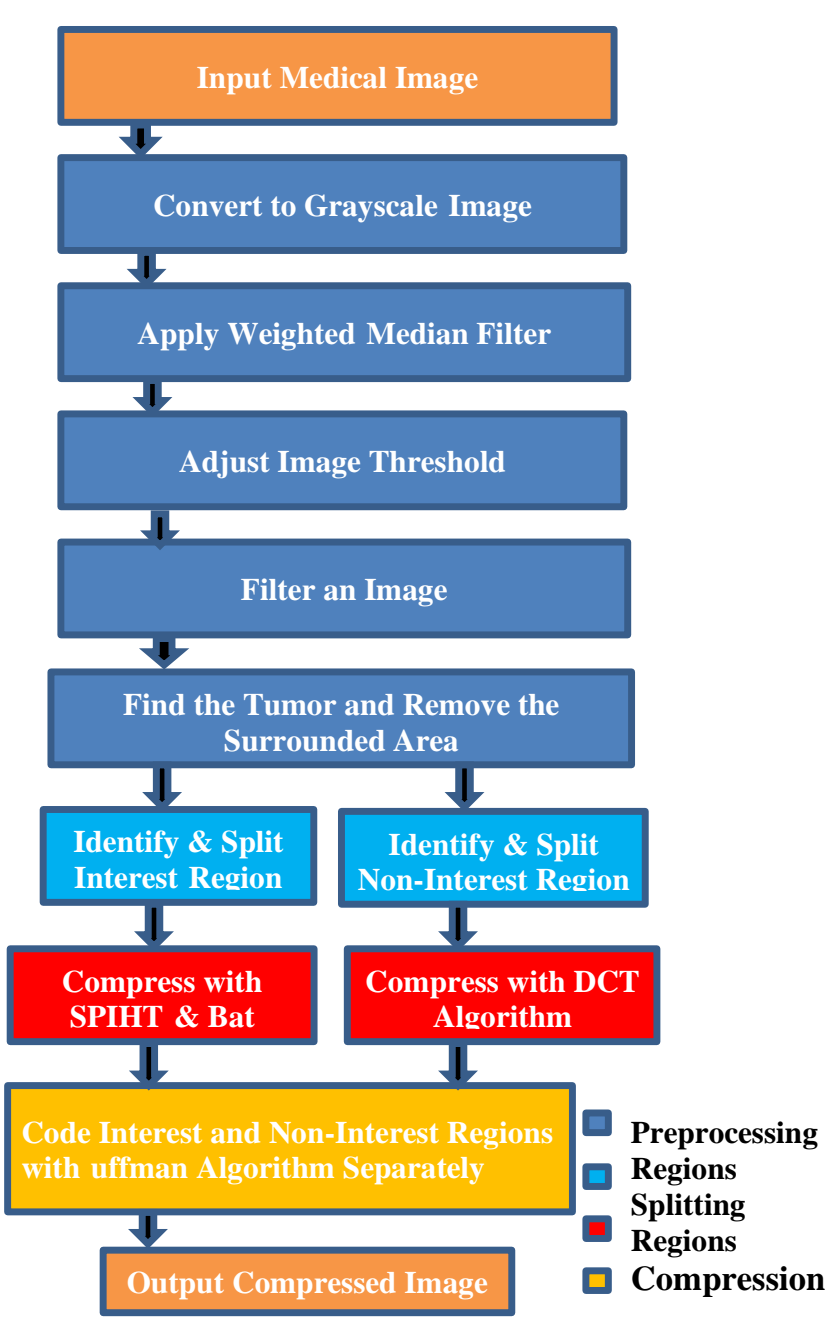

Fig 2: The overall framework of the proposed technique. 
There are several steps which depict the proposed hybrid compression technique:

Step 1: Convert the medical image from color image to grayscale image.

Step 2: Eliminate the noise by utilizing the weighted median filter.

Step 3: Binarize the filtered image (adjust the image threshold).

Step 4: Apply the median filter for decreasing the effect of noise on the image with threshold.

Step 5: Remove the surrounded area from the medical image to eliminate the unwanted area that appears as white color in skin cancer image.

Step 6: Identify and split the interest region from the noninterest region. Each unnecessary object (small) and does not relate to the main one should be eliminated. For instance, the widest white color object stills after the edge discovering in the skin cancer image since this object is considered as the interest region.

Step 7: Compress the interest region using the SPIHT and Bat inspired algorithms. The Bat estimates the required varying in threshold values to direct this particle in the space. The Bat can decrease the bit size of the image. So, image quality is enhanced. This inspired algorithm should be continued to obtain the optimal values of the threshold.

Step 8: Compress the non-interest region using the DCT technique.

Step 9: Apply Huffman coding algorithm to enlarge the compression ratio and improve compression performance to the compressed regions Separately (see Algorithm 2). This algorithm is faster for transmission purposes since it does not need a high level of computational complexity. Figure 3 illustrates the procedure of Huffman coding where the code is constructed from the left to the right and is written in reverse direction.

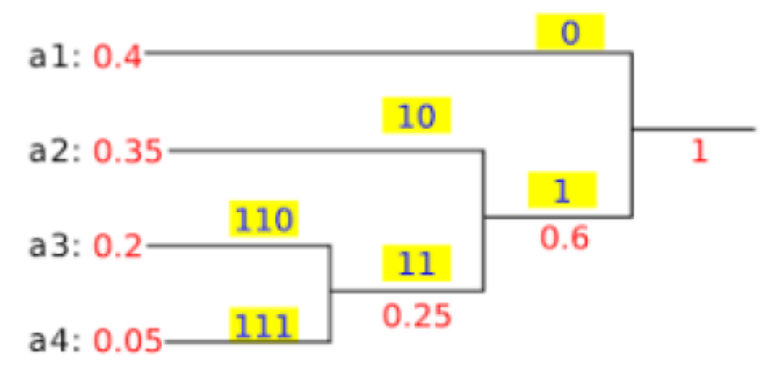

Fig 3: Huffman coding tree.

\begin{tabular}{|l|}
\hline Algorithm 2: Huffman encoding \\
\hline Input: A sorted list of one-node binary trees $\left(t_{l}, t_{2}, \ldots, t_{n}\right)$ \\
for alphabet $\left(S_{l}, \ldots, S_{n}\right)$ with frequencies $\left(W_{l}, \ldots, W_{n}\right)$. \\
\hline Output: A Huffman code with $n$ code words. \\
\hline Step 1: Build a binary tree where the leaves of the tree \\
are the symbols in the \\
alphabet. \\
Step 2: The edges of the tree are labeled by a 0 or 1. \\
Step 3: Derive the Huffman code from the Huffman tree. \\
\hline
\end{tabular}

\section{THE EXPERIMENTAL RESULTS}

In the proposed technique, the type of medical images that has been examined is skin cancer images of size $(512 \times 512)$. Table 1 shows three carefully selected skin cancer images, and their outputs from the pre-processing stage.

\section{TABLE (1): THE OBTAINED SKIN CANCER IMAGES AFTER} THE PREPROCESSING STAGE.

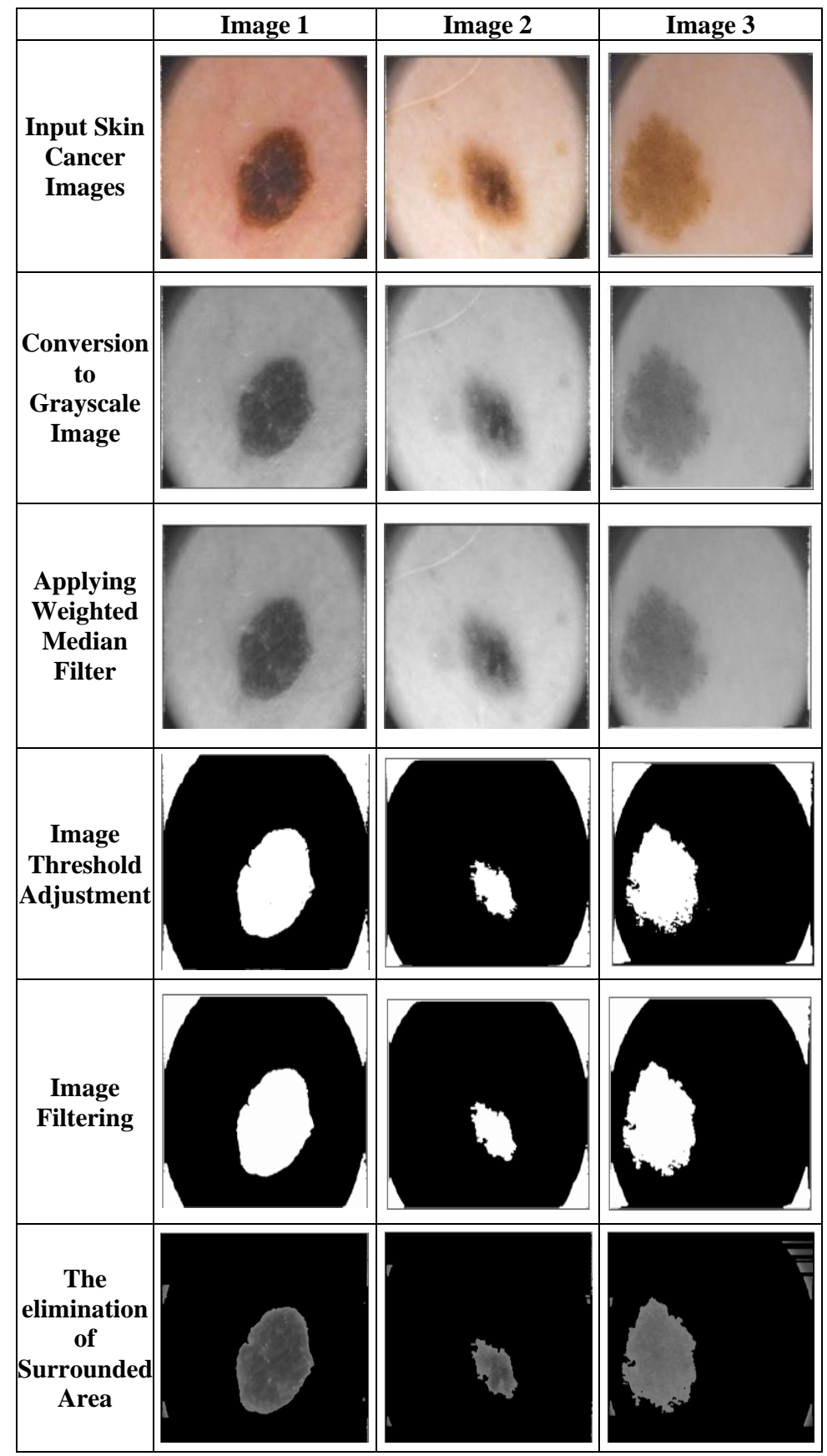

\section{TABLE (2): THE OBTAINED SKIN CANCER IMAGES AFTER THE REGIONS SPLITTING, COMPRESSION AND DECOMPRESSION STAGES.}

Table 2 indicates the resulted skin cancer images of applying the regions splitting, and regions decompression. The interest region images are approximately similar to the corresponding 
decompression images. Therefore, the processes of compression and decompression are successful.

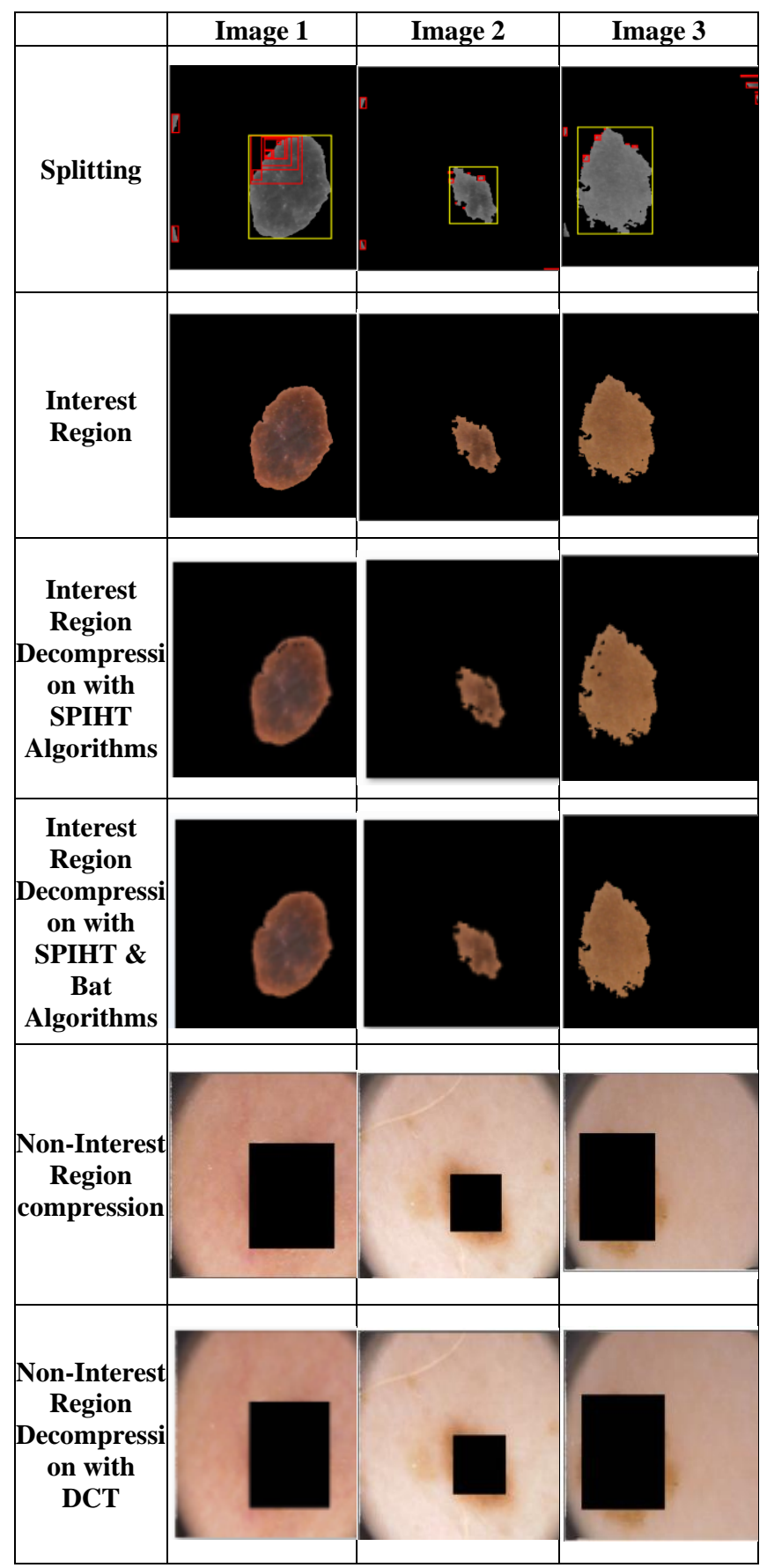

Mean Square Error (MSE) has been used to measure the likeness between the reconstructed and original skin cancer. The MSE can be formulated as follows:

$$
M S E=\frac{1}{N M} \sum_{i=0}^{N-1} \sum_{j=0}^{M-1}(X(i . j)-Y(i . j))^{2}
$$

A ratio of maximum value of image power to the noise power called Peak Signal to Noise Ratio (PSNR) which can be formulated as follows:

$$
P S N R=10 \log _{10} \frac{\left(2^{n}-1\right)^{2}}{M S E}
$$

A comparison between the proposed hybrid compression technique and SPIHT has been presented, and the proposed technique shows better results (see Table 3 ). These results indicate that the proposed technique has better PSNR values.

TABle (3): THE OBTAINEd PSNR VALUES.

\begin{tabular}{|l|c|c|c|c|c|c|}
\hline & \multicolumn{2}{|c|}{ Image 1 } & \multicolumn{2}{c|}{ Image 2 } & \multicolumn{2}{c|}{ Image 3 } \\
\hline & SPIHT & $\begin{array}{c}\text { SPIHT \& } \\
\text { Bat }\end{array}$ & SPIHT & $\begin{array}{c}\text { SPIHT \& } \\
\text { Bat }\end{array}$ & SPIHT & $\begin{array}{c}\text { SPIHT \& } \\
\text { Bat }\end{array}$ \\
\hline PSNR & 41.1157 & 60.1525 & 41.2527 & 61.4315 & 38.2864 & 53.1067 \\
\hline & \multicolumn{5}{|c|}{ DCT } & Image 3 \\
\hline PSNR & \multicolumn{2}{|c|}{ Image 1 } & \multicolumn{2}{|c|}{ Image 2 } & 29.9285 \\
\hline
\end{tabular}

\section{v. ConClusions}

For medical applications, an efficient technique based on SPIHT and Bat inspired algorithms has been proposed for skin cancer image compression with a low level of computational complexity. The obtained results of the proposed technique and the success of the architecture have been evaluated with PSNR criteria, and these results indicate that the proposed technique has better PSNR values. For increasing the compression performance, in the future, another inspired algorithm can be utilized with the SPIHT algorithm.

The proposed system passes all the randomness tests provided by NIST.

\section{REFERENCES}

[1] S. Ceklı and A. Akman, "An efficient SPIHT algorithm and system architecture for image compression," 2017 25th Signal Processing and Communications Applications Conference (SIU), Antalya, 2017, pp. 14.

[2] N.R. Rema, Binu Ani Oommen, P. Mythili, "Image Compression Using SPIHT with Modified Spatial Orientation Trees", Procedia Computer Science, Vol. 46, 2015, pp. 1732-1738.

[3] C. Kamargaonkar and M. Sharma, "Hybrid medical image compression method using SPIHT algorithm and Haar wavelet transform," 2016 International Conference on Electrical, Electronics, and Optimization Techniques (ICEEOT), Chennai, 2016, pp. 897-900.

[4] M. Sharma, C. Kamargaonkar and J. K. Rai, "Medical image sequence compression using fast block matching algorithm and SPIHT," 2016 3rd International Conference on Computing for Sustainable Global Development (INDIACom), New Delhi, 2016, pp. 1459-1463.

[5] B. Panjavamam and P. T. V. Bhuvaneswari, "Performance analysis of SPIHT algorithm for biomedical image transmission," 2017 Fourth International Conference on Signal Processing, Communication and Networking (ICSCN), Chennai, 2017, pp. 1-5.

[6] A. Kaveh, "Advances in met heuristic algorithms for optimal design of structures", A book, Springer, 2014.

[7] Z. Michalewicz and D. B. Fogel, "How to Solve It: Modern Heuristics", A book, Springer, 2004.

[8] Ahmed, H. and J. Glasgow, "Swarm intelligence: concepts, models and applications", School of Computing, Queens University, Technical Report, 2012.

[9] Tao Xiang, Jinyu Qu, Di Xiao, "Joint SPIHT compression and selective encryption", Applied Soft Computing, Vol. 21, 2014, pp. 159-170.

[10] Saif Saad, Fouad H. Awad, Ahmed S. Farhan, "Enhance the Hiding Image by Using Compression and Securing Techniques", Iraqi Journal for

Computers and Informatics, Vol. 43, 2017, pp. 14-16. 